\title{
NUMERICAL FLOW VISUALIZATION STUDIES IN INTERNAL COMBUSTION ENGINE
}

\author{
Lucky Anetor $^{1 *}$, Christopher Odetunde $^{2}$, Edward E. Osakue $^{3}$ \\ ${ }^{I}$ Department of Mechanical Engineering, Nigerian Defence Academy, Kaduna, Nigeria \\ ${ }^{2}$ Department of Aeronautics and Astronautics Engineering, Kwara State University, Ilorin, Nigeria \\ ${ }^{3}$ Department of Industrial Technology, Texas Southern University, Houston, Texas USA \\ E-mail:*anetor55@yahoo.com(Corresponding author)
}

\begin{abstract}
The KIVA-II code was used to simulate the effects of two different piston-bowl (re-entrant and bowl-in-piston) configurationsand two intake swirl levels (0.5 and 2.0) on the structure and evolution of the flow field at the top dead center position of an internal combustion engine. The results show that high-shear regions and the consequent turbulence production occurred near the bowl entrance around top dead center during the compression stroke. The regions were created before top dead center in both chambers by the interaction of swirl and squish. The mean radial and tangential velocities produced by the re-entrant bowl were found to be consistently higher than those generated by the bowl-in-piston configuration. It was found that the re-entrant bowl assembly generated 35\% more turbulence than the bow-in-piston design while the squish penetration for low intake swirl (0.5) was about $20 \%$ greater than for high intake swirl (2.0). The results from this study shows that after top dead center, high shear and reverse-squish flow occurs in both combustion chambers, however, they were more intense in the re-entrant bowl assembly. In view of the foregoing, it is recommended that the re-entrant bowl assembly be considered as the preferred choice in combustion chamber design.
\end{abstract}

Keywords: Engines, $\mathrm{NO}_{X}$, Turbulence, Length Scale, Swirl, Squish, Numerical Flow Visualization

\section{INTRODUCTION}

The fossil fuel reserves around the world are presently been consumed at an unsustainable rate. Moreover, various Environmental Protection Agencies (EPAs) have started promulgating more stringent emission control standards for Internal Combustion Engines (ICEs) and other forms of combustion devices - gas turbines, industrial burners, boilers etc. Another potentially harmful factor which is capable of aggravating the already deteriorating situation even further is the political differences which often manifest themselves regularly between major oil consumer nations of the West and the leading petroleum producing countries of the Middle East. These events have the potential to adversely impact the economic, energy and environmental security of the United States of America (USA). It is worth mentioning that on-road vehicles account for nearly 60 percent of the total USA oil consumption and more than 25 percent of the country's greenhouse gas emissions, the major contributor to climate change [1].

In order to ameliorate the consequences of the impact the issues mentioned above could have on the wellbeing of the United States of America, the USA Department of Energy established the Vehicle Technologies Office (VTO) which supports research and development (R\&D) and deployment of efficient and sustainable highway transportation technologiesthat will improve fuel economy and enable America to use less petroleum. It is envisaged that these technologies, which include plug-in electric vehicles (also known as EVs or electric cars), batteries, electric drive technologies, advanced combustion engines,lightweight materials and alternative fuels would increase Americans mobility and energy security, while lowering costs and reducing environmental impacts.

It has long been realized that fluid motion (turbulence) within the combustion chamber of an ICE play a very important role in controlling the combustion process [2 - 9]. The degree and details of this influence on combustion are still not very well understood. However, it can be safely assumed that the major roles of fluid mechanics in spark and compression ignition engines are first and foremost to prepare the mixture of fuel and air for combustion and secondly, to control flammability through large and small scale mixing. The mixture preparation in conventional spark ignition engine is achieved mainly by the induction system, which generates the bulk motion and the subsequent turbulence required to mix both air and fuel prior to ignition. In diesel engines, the induction system (for example, swirl, and tumble) and chamber geometry (for example, squish) control the air motion. The flow pattern also affects the rate of heat transfer to the cylinder walls, turbulent transport of mass, momentum and energy. Extensive experimental and computational studies are presently been carried out with the aim ofdemonstratingthe effectiveness and applications of these ideas in the area of internal combustion engine research [10 17].

Effective usage of fuel is greatly dependent upon engine efficiency, which in turn depends to a large extent on how fuel is burned within the cylinders of an engine. Higher 
pressures and temperatures within the cylinder lead to increased fuel economy. However, they also make controlling the combustion process very difficult. Inadequatecontrol and incomplete combustion can cause higher levels of emissions and lower engine efficiencies.

In order to optimize combustion processes, engine designers have traditionally relied on build, test, analyze the results and modify method. This iterative process is painfully slow, costly and does not lend itself to identifying the optimal engine design specifications. In view of these problems, the engineers at Los Alamos National Laboratory developed the KIVAfamily codes, an advanced computational fluid dynamics (CFD) modeling code that accurately simulatesthermal engine in-cylinder processes.

\section{OBJECTIVE OF THE PRESENT STUDY}

From the foregoing and the references cited, we can infer that detailed knowledge of the mechanism(s) of fluid motion (swirl-squish interaction) in internal combustion engine and the consequent turbulence generated is still lacking. Since experimentation is very expensive and time consuming and could not possibly provide the level of flow visualization required for studying in-cylinder processes, we decided to use the KIVA-II code to study the flow fields in two combustion chambers, namely; bowl-in-piston and the reentrant piston bowl geometries.

\section{NUMERICAL MODELING}

Computational fluid dynamics has become an established tool for studying and designing real-world combustion systems. Multidimensional models have proven their value in reducing the need for actual experimentation, the benefit of which has been a reduction in product development time and cost.

KIVA-II code is a transient, three-dimensional, multiphase, multicomponent numerical simulation code for the analysis of chemically reacting flows with sprays. The code uses an Arbitrary Lagrangian-Eulerian (ALE) methodology on a staggered grid and discretizes space using the finite volume method. It uses an implicit time-advancement with the exception of the advection terms that are cast in an explicit but second-order monotonicity-preserving manner. Furthermore, the convection calculations can be sub-cycled in the desired regions in order to avoid restricting the time step due to Courant conditions.

Brief descriptions of the numerical models used for this study are presented below. Detailed treatment of these models can be found in [16 - 17].

\subsection{Turbulence Model}

Two models are available to represent the effects of turbulence, namely the $k-\varepsilon$ and the sub-grid scale (SGS) models. The standard version of the $k-\varepsilon$ turbulence model, modified to include volumetric expansion effects was used in the present work. It is pertinent to point out that the subgrid scale model reduces to the $k-\varepsilon$ turbulence model near walls where all turbulence length scales are too small to be resolved by the computational mesh. Boundary layer drag and wall heat transfer are calculated by matching to a modified turbulent law-of-the-wall.

\subsection{The Governing Equations}

The equations of motion for the fluid phase and boundary conditions are given in this section. For compactness these equations are written in vector notations. The unit vectors in the $x, y$ andzdirections are denoted by $\vec{i}, \vec{j}$ and $\vec{k}$ respectively.

The position vector $\vec{r}$ is defined as:

$$
\vec{r}=x \vec{\imath}+y \vec{j}+z \vec{k}
$$

the vector (Del) operator $\nabla$ is given by:

$$
\nabla=\vec{\imath} \frac{\partial}{\partial x}+\vec{j} \frac{\partial}{\partial y}+\vec{k} \frac{\partial}{\partial z}
$$

and the fluid velocity vector $\vec{u}$ is given by:

$$
\vec{u}=u(x, y, z, t) \vec{\imath}+v(x, y, z, t) \vec{\jmath}+w(x, y, z, t) \vec{k}
$$

wheret is the time.

\subsection{The Fluid Phase}

The KIVA-II equations can be used to solve for both laminar and turbulent flows. The mass, momentum and energy equations for the two cases differ primarily in the form and magnitude of the transport coefficients (that is, viscosity, thermal conductivity and species diffusivity), which are much larger in the turbulent case because of the additional transport caused by turbulent fluctuations. In the turbulent regime, the transport coefficients are derived from a turbulent diffusivity that depends on the turbulent kinetic energy, $k$ and its dissipation rate, $\varepsilon$.

\subsection{Continuity Equation}

The continuity equation for species mis:

$$
\frac{\partial \rho_{m}}{\partial t}+\nabla \cdot\left(\rho_{m} \vec{u}\right)=\nabla \cdot\left[\rho D \nabla\left(\frac{\rho_{m}}{\rho}\right)\right]+\dot{\rho}_{m}^{c}+\dot{\rho}^{s} \delta_{m 1}
$$

where $\rho_{m}$ is the mass density of species $m, \rho$, the total mass density and $\vec{u}$, the fluid velocity. Fick's Law is assumed for diffusion processes, with a single diffusion coefficient, $D$. The equation for $D$ is given below. Species 1 is that for which the spray droplets are composed, and $\delta_{m 1}$ is the Dirac delta function. By summing Equation (1) over all species, we obtain the total fluid density equation, thus:

$$
\frac{\partial \rho}{\partial t}+\nabla \cdot(\rho \vec{u})=\dot{\rho}^{s}
$$

Since mass is conserved in chemical reactions. Where $\dot{\rho}^{s}$ is the spray density. In the present work, 


$$
\dot{\rho}^{s}=0 .
$$

\subsection{Momentum Equation}

The momentum equation for the fluid mixture is given by:

$$
\begin{aligned}
\frac{\partial(\rho \vec{u})}{\partial t}+\nabla \cdot(\rho \vec{u} \vec{u}) & \\
= & -\frac{1}{\alpha^{2}} \nabla p-A_{0} \nabla\left(\frac{2}{3} \rho k\right)+\nabla \cdot \tilde{\sigma} \\
& +\vec{F}^{s}+\rho \vec{g}
\end{aligned}
$$

where $p$ is the fluid pressure. The dimensionless quantity $\alpha$ is used in conjunction with the Pressure Gradient Scaling (PGS) Method, [16] and [17]. This is a method for enhancing computational efficiency in low Mach number, $M a$ flows where the pressure is nearly uniform.

In Equation (3) the quantity $A_{0}$ is zero in laminar calculations and unity when one of the turbulence models is invoked.

The viscous stress tensor, $\tilde{\sigma}$ is Newtonian in form, therefore;

$$
\tilde{\sigma}=\mu_{e f f}\left[\nabla \vec{u}+(\nabla \vec{u})^{T}\right]+\lambda \nabla \cdot \vec{u} \tilde{I}
$$

The first and second coefficients of viscosity, $\mu_{\text {eff }}$ and $\lambda$ are defined below. The superscript $T$ denotes the transpose and $\tilde{I}$ is the unit dyadic. $\vec{F}^{s}$ is the rate of momentum gain per unit volume due to the spray. The specific body force, $\vec{g}$ is assumed constant.

\subsection{Turbulent Kinetic Energy and Dissipation}

\section{Models}

When one of the turbulence models is used, $A_{0}=1$, two additional transport equations are solved for the turbulence kinetic energy, $k$ and its dissipation rate, $\varepsilon$ thus:

$$
\begin{aligned}
\frac{\partial(\rho k)}{\partial t}+\nabla \cdot(\rho \vec{u} k) & \\
= & -\frac{2}{3} \rho k \nabla \cdot \vec{u}+\widetilde{\sigma}: \nabla \vec{u}+\nabla \\
\cdot & {\left[\left(\frac{\mu_{e f f}}{P r_{k}}\right) \nabla k\right]-\rho \varepsilon+\dot{W}^{s} }
\end{aligned}
$$

and;

$$
\begin{aligned}
\frac{\partial(\rho \varepsilon)}{\partial t}+\nabla \cdot(\rho \vec{u} \varepsilon) & \\
= & -\left(\frac{2}{3} c_{\varepsilon_{1}}-c_{\varepsilon_{3}}\right) \rho \varepsilon \nabla \cdot \vec{u}+\nabla \\
& \cdot\left[\left(\frac{\mu_{e f f}}{P r_{\varepsilon}}\right) \nabla \varepsilon\right] \\
+ & \frac{\varepsilon}{k}\left[c_{\varepsilon_{1}} \tilde{\sigma}: \nabla \vec{u}-c_{\varepsilon_{2}} \rho \varepsilon+c_{s} \dot{W}^{s}\right]
\end{aligned}
$$

Equations (5) and (6) are the standard $k-\varepsilon$ equations with some added terms. The source term

$\left(\frac{2}{3} c_{\varepsilon_{1}}-c_{\varepsilon_{3}}\right) \rho \varepsilon \nabla \cdot \vec{u}$ in the $\varepsilon$ equation accounts for length scale changes when there is velocity dilation. Source terms involving the quantity $\dot{W}^{s}$ arise due to interaction with spray. In the present study, $\left(\dot{W}^{s}=0\right)$.

The quantities, $c_{\varepsilon_{1}}, c_{\varepsilon_{2}}, c_{\varepsilon_{3}}, c_{s}, P r_{k}$ and $P r_{\varepsilon}$ are constants whose values are $1.44,1.92,-1.0,1.5,1.0$ and 1.3 respectively. They are determined from experiments and some theoretical considerations.

The physical meaning of the various terms in Equation (5) is as follows; the term $\nabla \cdot(\rho \vec{u} k)$ simply represents convection of the turbulence by the resolved velocity field. The term $-2 / 3(\rho k \nabla \cdot \vec{u})$ is a compressibility term that represents the turbulent analogy of $P d V$ work. The term $\widetilde{\sigma}: \nabla \vec{u}$ represents the production of turbulence by shear in the resolved velocity field and $\nabla \cdot\left[\left(\frac{\mu_{\text {eff }}}{P r_{k}}\right) \nabla k\right]$ represents the self-diffusion of the turbulence with a diffusivity of $\mu_{e f f} / \rho$. The term $-\rho \varepsilon$ represents the decay of turbulence into thermal energy.

The transport coefficients in Equations (1) to (6) are:

$$
\begin{gathered}
\mu_{e f f}=\left(1-A_{0}\right) \rho v_{0}+\mu_{\text {air }}+A_{0} c_{\mu} \frac{k^{2}}{\varepsilon} \\
\lambda=A_{3} \mu_{e f f}
\end{gathered}
$$

and,

$$
D=\frac{\mu_{e f f}}{\rho S c}
$$

The diffusivity $v_{0}$ is an input constant, and $c_{\mu}$ is an empirical constant with a standard value of 0.09 . A Sutherland formula is used for $\mu_{\text {air }}$, thus:

$$
\mu_{\text {air }}=\frac{A_{1} T^{3 / 2}}{T-A_{2}}
$$

where $A_{1}$ and $A_{2}$ are constants. The constant $A_{3}$ is taken to be $-2 / 3$ in calculations of turbulent flow, but can be arbitrarily specified in laminar flows. The Prandtl and Schmidt numbers, Prand $S c$ are input constants.

\section{COMPUTATIONAL DOMAIN AND MESH}

The computational domain over which solutions of the differential equations described in section 3 are required is that lying between the cylinder surfaces, cylinder head and piston face. In computational fluid dynamics parlance, these surfaces are referred to as walls. In reciprocating engines two of these walls move - the piston face moves continuously in time and the valves move intermittently. If a finite-difference computational mesh which is fixed in space is used, much of the grid will not be utilized during parts of the piston cycle. A mesh which does not translate with the piston motion is therefore very wasteful of both computer time and storage memory. This issuewas resolved by defining a mesh which moves with the motion of the piston such that the whole mesh is always confined between the cylinder and piston head. Various ways of accomplishing this have been proposed, but the Arbitrary Lagrangian-Eulerian (ALE) technique $[9,10]$ has been found to be the most suitable. This method was used in the present work. 


\subsection{Boundary Conditions}

In order to completely specify a numerical problem it is necessary to supply the conditions at the boundaries of the solution domain for the variables. These conditions are usually obtained by either specifying the value of the dependent variable at the boundary, or the value of the associated flux, or a relation between the two. The specifications of the relevant boundary conditions employed in this work also follow these basic rules.

\subsection{Walls - Velocities and Pressure}

Velocity boundary conditions on rigid walls are introduced either by imposing the value of the velocity on the walls or the wall stress, $\tilde{\sigma}_{w}=\tilde{\sigma} \cdot \vec{n}$, where $\vec{n}$ is the unit normal to the wall. On no slip walls, the gas velocity is set to the wall velocity, thus:

$$
\vec{u}=w_{\text {wall }} \vec{k}
$$

where the wall (piston) is assumed to be moving with speed $w_{\text {wall }}$ in the $z$ direction. The wall stress is then determined implicitly through Equation (4). On free-slip and turbulent law-of-the-wall boundaries the normal gas velocity was set equal to the normal wall velocity as follows:

$$
\vec{u} \cdot \vec{n}=w_{\text {wall }} \vec{k} \cdot \vec{n}
$$

and the two tangential components of $\tilde{\sigma}_{w}$ are explicitly specified. For free-slip walls the tangential components of $\tilde{\sigma}_{w}$ are zero.

\subsection{Turbulent Law-of-the-Wall Conditions}

For turbulent law-of-the-wall conditions the tangential components were determined by matching to a logarithmic profile, thus:

$$
\frac{v}{u^{*}}=\left\{\begin{array}{c}
\frac{1}{\kappa} \ln \left(c_{l_{w}} \xi^{7 / 8}\right)+B, \quad \text { for } \xi>R_{c} \\
\xi^{\frac{1}{2}}, \quad \text { for } \xi<R_{c}
\end{array}\right.
$$

where

$$
\xi=\frac{\rho y_{p} v}{\mu_{\text {air }}(T)}
$$

is the Reynolds number based on the gas velocity relative to the wall, which is given by:

$$
v=\left|\vec{u}-w_{\text {wall }} \vec{k}\right|
$$

which is evaluated a distance $y_{p}$ from the wall and $u^{*}$ is the shear speed, which is related to the tangential components of the wall stress by:

$$
\tilde{\sigma}_{w}-\left(\tilde{\sigma}_{w} \cdot \vec{n}\right) \vec{n}=\rho\left(u^{*}\right)^{2} \frac{\vec{v}}{v}
$$

where,

$$
\vec{v}=\vec{u}-w_{\text {wall }} \vec{k}
$$

In Equations (14) and (16) it is assumed that $y_{p}$ is small enough to be in the logarithmic region or the laminar sublayer region of the turbulent boundary layer. The Reynolds number $R_{c}$ defines the boundary between these two regions. The constants $\kappa, c_{l_{w}}, R_{c}$ and $B$ in Equation (13) are related to the $k-\varepsilon$ turbulence model constants by:

$$
\kappa=\sqrt{c_{\mu}^{1 / 2}\left(c_{\varepsilon_{2}}-c_{\varepsilon_{1}}\right) P r_{\varepsilon}}
$$

and,

$$
B=R_{c}^{1 / 2}-\frac{1}{\kappa} \ln \left(c_{l_{w}} R_{c}^{7 / 8}\right)
$$

For commonly accepted values of the $k-\varepsilon$ turbulence model constants, $B=5.5$ and $c_{l_{w}}=-0.15$,we obtain, $\kappa=0.4327$ and $R_{c}=114$. When the velocities are specified at a boundary, the pressure need not be specified there.

\subsection{Turbulent Kinetic Energy and Dissipation Rate}

In calculations of the turbulent flow, boundary conditions are also required for the turbulent kinetic energy, $k$ and its dissipation rate, $\varepsilon$. Recall that the standard $k-\varepsilon$ turbulence model is not valid in the buffer or the linear sub-layer region of the turbulent boundary layer because the local Reynolds number $\bar{\rho} k^{2} / \mu \varepsilon$ is low in this region. Consequently, boundary conditions for Equations (5) and (6) as well as the ensemble-averaged conservation equations of $k$ and $\varepsilon$ are applied at a distance $y_{p}$ away from the wall in the fully turbulent region (the log-law layer) where the local Reynolds number is sufficiently high. The boundary conditions applied at such a location away from the wall are referred to as wall functions.

In the present work, the wall functions are given as follows; since the shear stress near the wall is nearly constant, the wall function for turbulent kinetic energy, $k$ is given by:

$$
\nabla k \cdot \vec{n}=0
$$

and, in the log-law layer, the production of turbulence is approximately equal to its rate of dissipation (local isotropy). Therefore, the wall functions for $\varepsilon$ is approximated by:

$$
\varepsilon=c_{\mu_{\varepsilon}} \frac{k^{3 / 2}}{y_{p}}
$$

where $k$ and $\varepsilon$ are evaluated at a distance $y_{p}$ from the wall, and,

$$
c_{\mu_{\varepsilon}}=\left[\frac{c_{\mu}}{\operatorname{Pr}_{\varepsilon}\left(c_{\varepsilon_{2}}-c_{\varepsilon_{1}}\right)}\right]^{1 / 2}
$$

\section{THE NUMERICAL SCHEME}

The finite-difference approximations to the governing equations of sections 3 and 4 were solved with the KIVA-II code. The equations were discretized both in space and time. 


\subsection{Temporal Differencing}

The temporal differencing is performed with respect to a sequence of discrete timest $t^{n}$, where, $(n=0,1,2, \ldots)$. The time interval $\Delta t^{n}=t^{n+1}-t^{n}$ is the time-step, and the integer $n$ is the cycle number. In view of the foregoing, the difference approximation to the derivative $\partial Q / \partial t$ is the firstorder expression $\left(Q^{n+1}-Q^{n}\right) / \Delta t^{n}$. In the KIVA-II code, a cycle is performed in three stages or phases, namely $A, B$ and $C$.Phases $A$ and $B$ together constitute a Lagrangian calculation in which computational cells move with the fluid. In phase $C$ the flow field is frozen and rezoned or remapped onto a new computational mesh.

\subsection{Spatial Differencing}

The spatial differencing is based on the $A L E$ method, which in three dimensions uses a mesh made up of arbitrary hexahedrons. Spatial difference approximations are performed by integrating the differential term of interest over the volume of a typical cell. Volume integrals of gradient or divergence terms are converted into surface area integrals using the divergence theorem. Volume and surface integrals were assumed to be uniform within cells or on cell surfaces. Therefore, area integrals over surfaces of cells become sum over cell faces or sub-faces, thus:

$$
\iint \vec{F} \cdot d \vec{S} \rightarrow \sum_{a} \vec{F}_{a} \cdot \vec{S}_{a}
$$

\subsection{Solution Procedure}

The solution method is patterned after the Semi-Implicit Method for Pressure-Linked Equations(SIMPLE) procedure [18] with individual equations solved using the conjugate residual method.

\subsection{Accuracy Conditions and Automatic Time-step}

\section{Control}

The time-steps $\Delta t$ and convection time-step $\Delta t_{c}$ are selected automatically at the beginning of each calculation cycle. Because diffusion terms are differenced implicitly and convection terms are subcycled, there are no stability restrictions on $\Delta t$.However, there are several accuracy conditions upon which the automatic selection of $\Delta t$ is based $[9,10]$. The convection time-step $\Delta t_{c}$ must satisfy the Courant condition for stability.

The first accuracy condition on $\Delta t$ is that:

$$
\left|\frac{D \vec{u}}{D t}\right| \Delta t^{2}<f_{a} \Delta x
$$

where $f_{a}$ is some positive real number of order unity and $\Delta x$ is an average cell dimension. This condition arises because terms of order higher than $\Delta t$ are ignored in the phase $B$ cell vertex positions. The default value of $f_{a}=0.5$ was used in the present work.

The second accuracy condition on $\Delta t$ is that:

$$
|\lambda| \Delta t<f_{r}
$$

where $f_{r}$ is of order unity and $\lambda$ is an eigenvalue of the rate of strain tensor. This criterion limits the amount of cell distortion that can occur due to mesh movement in the Lagrangian phase. When cells become too distorted, the spatial accuracy of the difference approximations suffers. Using $f_{r}=1 / \sqrt{3}$ gave sufficient accuracy in the calculations reported in the present study.

Two other accuracy criteria for $\Delta t$ are obtained from the need to couple accurately the flow field and source terms due to chemical heat release and mass and energy exchange with spray. These criteria were not relevant in the present work.

The convection time-step $\Delta t_{c}$ is based on the Courant stability condition. In a rectangular mesh, this condition is given by:

$$
\Delta t_{c} \leq \min \left(\frac{\Delta x}{\left|u-b_{x}\right|}, \frac{\Delta y}{\left|v-b_{y}\right|}, \frac{\Delta z}{\left|w-b_{z}\right|}\right)
$$

Where $b_{x}, b_{y}$ and $b_{z}$ are the components of the grid velocity $\vec{b}$. Constraint (Equation (25)) limits the magnitude of the flux volume in any direction to a value less than the cell volume. To generalize this to an arbitrary mesh, it is natural to replace Equation (25) by the similar criterion:

$$
\Delta t_{c}^{n} \leq \min _{\alpha}\left(\frac{V_{i j k}}{\delta V_{\alpha}}\right) \cdot \Delta t_{c}^{n-1}
$$

where the $\delta V_{\alpha}$ are the flux volumes calculated for $\operatorname{cell}(i, j, k)$ using time-step $\Delta t_{c}^{n-1}$. In practice, for accuracy the time-step determined from Equation (26) is normally reduced by a factor, $f_{\text {conv }}=0.2$, this practice was also adopted in the present work.

\section{MODELING APPROACH}

The first stage in the theoretical study was to obtain the various computational meshes for the relevant combustion chamber geometries. These were generated using the supplied parametric mesh generator. The piston bowls were modeled as described below.

\subsection{Re-entrant Bowl Piston}

Figures 2 and 3 show the computational meshes for the reentrant and bowl-in-piston combustion chamber designs at 25 degree $(B T D C)$ and at the $T D C$ positions respectively. The reentrant bowl was modeled using $\mathrm{a} 9 \times 11 \times 10$ (radial $\times$ axial $\times$ circumferential mesh. The region above the piston was modeled with a mesh that had 21 cells in the radial direction, while the number of planes in the axial direction varies from 20 at inlet valve closure to 3 at top dead center(TDC).

\subsection{Bowl-in-Piston}

The bowl-in-piston combustion chamber was modeled using an $18 \times 13 \times 10($ radial $\times$ axial $\times$ circumferential $)$ mesh. The region above the piston was modeled with a mesh 
having 27 cells in the radial direction, while the number of planes in the axial direction varies from 20 at inlet valve closure to 4 at top dead center.

As the piston move upwards, the axial dimension of cells above the piston face decreases. Each time this axial dimension becomes less than a small predetermined value (in our studies this value was taken to be the cell height at top dead center position), one plane of cells above the piston is deleted and the flow field is remapped onto the new mesh. This procedure improves computational efficiency by maximizing the cell axial dimension and at the same time reducing the number of redundant cells. Conversely, the deleted planes are restored after the top dead center (ATDC) position when the piston is on its downward stroke. Cells lying within the piston bowls simply translate with the piston motion.

\subsection{Initial Conditions}

The calculations were initialized at 60 -degree $B T D C$ and computations were terminated at 20- degree $A T D C$. The initial temperature and pressure were set to300 $\mathrm{K}$ and 1.0 barrespectively. The initial velocity distribution was assumed to vary linearly between the piston and the cylinder head, being zero at the cylinder head and equal to the piston speed at the piston head.

At the boundaries of the computational domain, heat and momentum losses were calculated by assuming that boundary layers are turbulent and matching to a law-of-thewall velocity profile. The details can be found in $[9,10]$.

The initial swirl velocities $w(r)$ are related to the distance $r$ from the cylinder axis by:

$$
w(r)=U_{t} J_{1}\left(\lambda \cdot \frac{r}{R}\right)
$$

where $J_{1}$ is the first order Bessel Function, $R$ is the cylinder radius, and $\lambda$ is a constant with a value of 3.11. The choice of this profile was informed by the experimental measurements of swirling flows in cylinders [19].

The value of $U_{t}$ is calculated from:

$$
U_{t}=\frac{(S R) \cdot \Omega \cdot R \cdot \lambda}{4 \cdot J_{2}(\lambda)}
$$

where $S R$ is the swirl ratio, $\Omega$ is the engine angular velocity in radians per second, that is, $(\Omega=2 \pi \cdot \mathrm{RPM} / 60)$, RPMis engine speed in revolutions per minute and $J_{2}$ is the Bessel Function of order 2. This value of $U_{t}$ gives the same angular momentum as a solid body swirl with angular velocity, $(S R$. $\Omega$.

The initial turbulent kinetic energy was assumed to be uniform and equal to $10 \%$ of the square of the mean piston speed, while the initial turbulent length scale was assumed to be $40 \%$ of the distance from the each cell to the closest solid boundary.

\subsection{Numerical Accuracy}

It is well known that the quality of the solutions generated by computational fluid dynamics codes can be seriously degraded by numerical or false diffusion. The interpolated donor cell differencing scheme was used for the calculations presented in this study. It has the advantage of greater accuracy (second order versus first order) and smaller numerical diffusion over the upwind differencing scheme. As such, the Reynolds number at which the numerical and physical diffusive fluxes are equal is somewhat greater than 2.

In order to investigate possible degradation due to numerical diffusion and grid independence of the predicted solutions, computations were performed with grids of various densities and time-steps. The mesh designs stated above produced results which were grid-resolution independent. A computational time-step of $1.04167 \times 10^{-4}$ secondand smaller was found to produce stable results.

\section{NUMERICAL RESULTS}

The KIVA-II code was used to study/visualize the flow patterns of two different combustion chamber designs. The sample calculations represent typical operations of the compression, power and exhaust strokes of internal combustion engines. On the intake stroke, pure air is drawn into the cylinder through four tangentially placed inlet ports, causing the air to swirl uniformly about the axis of the cylinder. The combustion model embodied in the KIVA-II was disabled, hence giving rise to cold flow simulations. Apart from switching-off the combustion model, all other engine operating cycles, namely; compression, power and exhaust strokes were simulated. These calculations are for intake swirl levels 0.5 and 2.0 and percentage squish of 80 , for both the re-entrant and bowl-in-piston chamber configurations. The details of the operating parameters of the rapid intake and compression engineand the two piston configurations are shown in Table 1 and Fig.1.

Table 1. Rapid intake and compression machine operating parameters.

\begin{tabular}{|l|c|}
\hline $\begin{array}{l}\text { Bore (Engine Cylinder } \\
\text { Diameter) }\end{array}$ & $100.0 \mathrm{~mm}$ \\
\hline Stroke Length & $100.0 \mathrm{~mm}$ \\
\hline Compression Ratio & $8: 1$ \\
\hline Initial Pressure & $1.01325 \mathrm{MPa}$ \\
\hline Bowl-In-Piston Squish Area & $80 \%$ \\
\hline Re-entrant Piston Squish Area & $80 \%$ \\
\hline Intake Port Opens & 24 degree $B T D C$ \\
\hline Port Closes & 60 degree $B T D C$ \\
\hline Driving Cylinder Pressure & $900 \mathrm{kPa}$ \\
\hline Simulated Engine Revolution & $1000 \mathrm{rpm}$ \\
\hline
\end{tabular}




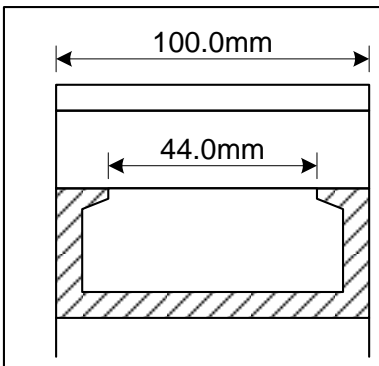

(a) Re-entrant bowl

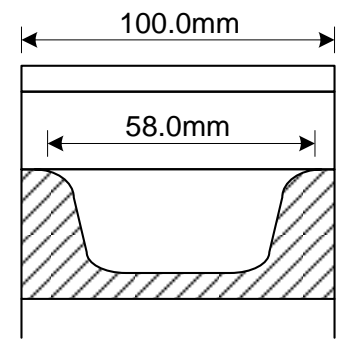

(b) Bowl-in-piston
Fig. 1: Re-entrant and Bowl-in-piston configurations.
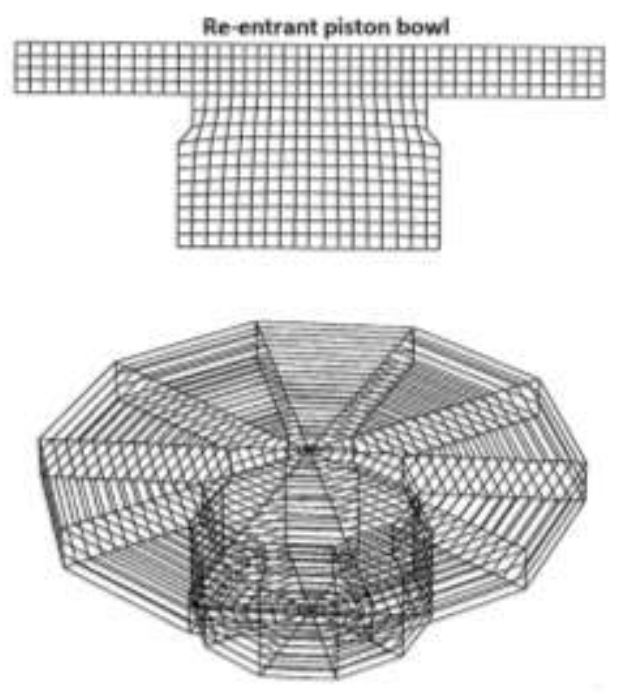

Fig. 2: Finite volume mesh at 25 degree $B T D C$.
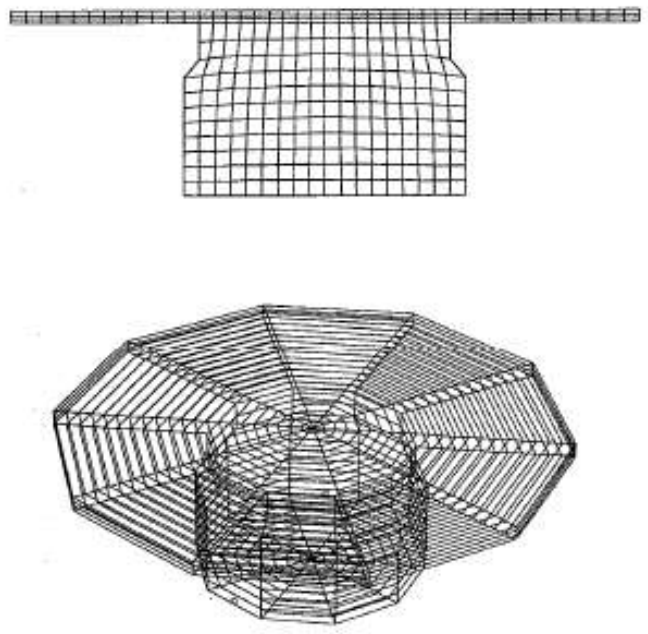

The flow quantities that were studied/visualized in the present work were the mean radial and tangential velocities and various turbulence parameters (turbulence intensity and length scale). These turbulence parameters were selected because various turbulent flame speed models/correlations and most qualitative descriptions of flame structure are based on them, [20] and [21]. The computational meshes of these pistons are shown in Figs.2and 3. The radial and tangential velocity components and various turbulence parameters emanating from these combustion chamber configurations were used as the basis for evaluating the combustion chamber effectiveness. The velocities shown in the present work were averaged in planes between the piston crown and cylinder head.
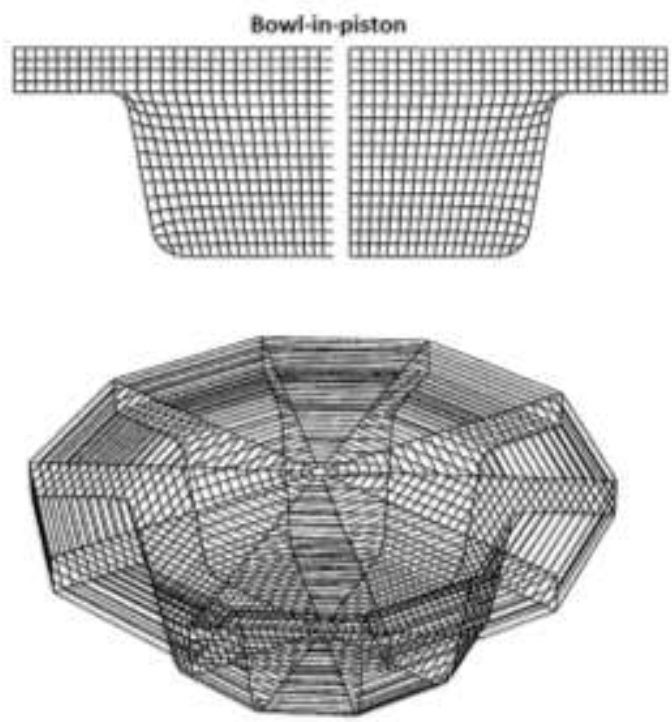

Fig. 3: Finite volume mesh at $T D C$.

\subsection{Discussion of the Computed Flow Fields}

A challenging aspect in studying the motion of charge flow in internal combustion engines is rooted in our inability to visualize the complex flow fields because combustion chambers are fabricated with opaque materials. As a result of this our ability to explain the causes of many flow features and different flow field properties become severely limited. These limitations are avoided when appropriate computational fluid dynamic code is used to simulate incylinder flow fields. In the present work, the velocity vector plots and contour maps of scalar variables in selected planes 
that slice through the three-dimensional flow field are presented. The velocity vector plots show the projections of the velocity vectors onto selected planes. By juxtaposing several such velocity plots, a reasonable understanding of the complex three-dimensional flow-field can be developed. Figs. 4 to 10 show a sample of the various flow fields generated.

\subsection{Velocity Vector Fields}

Some of the velocity fields generated by the re-entrant and bowl-in-piston combustion chambers from 25 degree $B T D C$ to 15 degree $A T D C$ are shown in Figs. 4 to 7 . From these figures, it can be observed that strong squish motions are generated by the re-entrant bowl configuration during compression stroke. These figures also show that, in the absence of swirl (low swirl 0.5), the strong radially inward flow out of the squish region at the bowl circumference sets up a torroidal flow within the bowl as expected. When swirl is present, however, the squish and swirl flows interact forcing a redistribution of flow's angular momentum. The centrifugal forces resulting from the intake swirl oppose the radially inward squish flow, causing it to turn into the bowl earlier. The resulting vortex flow in the bowl then rotates in opposite direction to the vortex in the low swirl case.

It is also evident from the figures presented that, apart from the short squish penetration observed in the re-entrant bowl for high intake swirl levels, the resulting squish attaches itself to the bowl wall just below the lip. This re-attachment phenomena is not particularly suitable for global turbulence generation, however, it only promotes localized high shear velocities around the lip of the bowl. The figures alsoshow that because of the short penetration range, there is hardly any interaction between the main flow and squish motion, whereas a high level of interaction is evident between the main flow and squish for the case of low or no intake swirl. A more serious drawback of this type of flow is that, the fuel particles could become separated from the air, due to the fuel particles inability (inertia) to follow such an abrupt change in air flow pattern. The evidence of this could be seen as film of fuel deposition both on the wall and at the bottom of the cup. However, because of the somewhat narrow channel created by the front of the squish motion, the main stream fluid, flows past in a jet-like fashion and hence generates some turbulence.

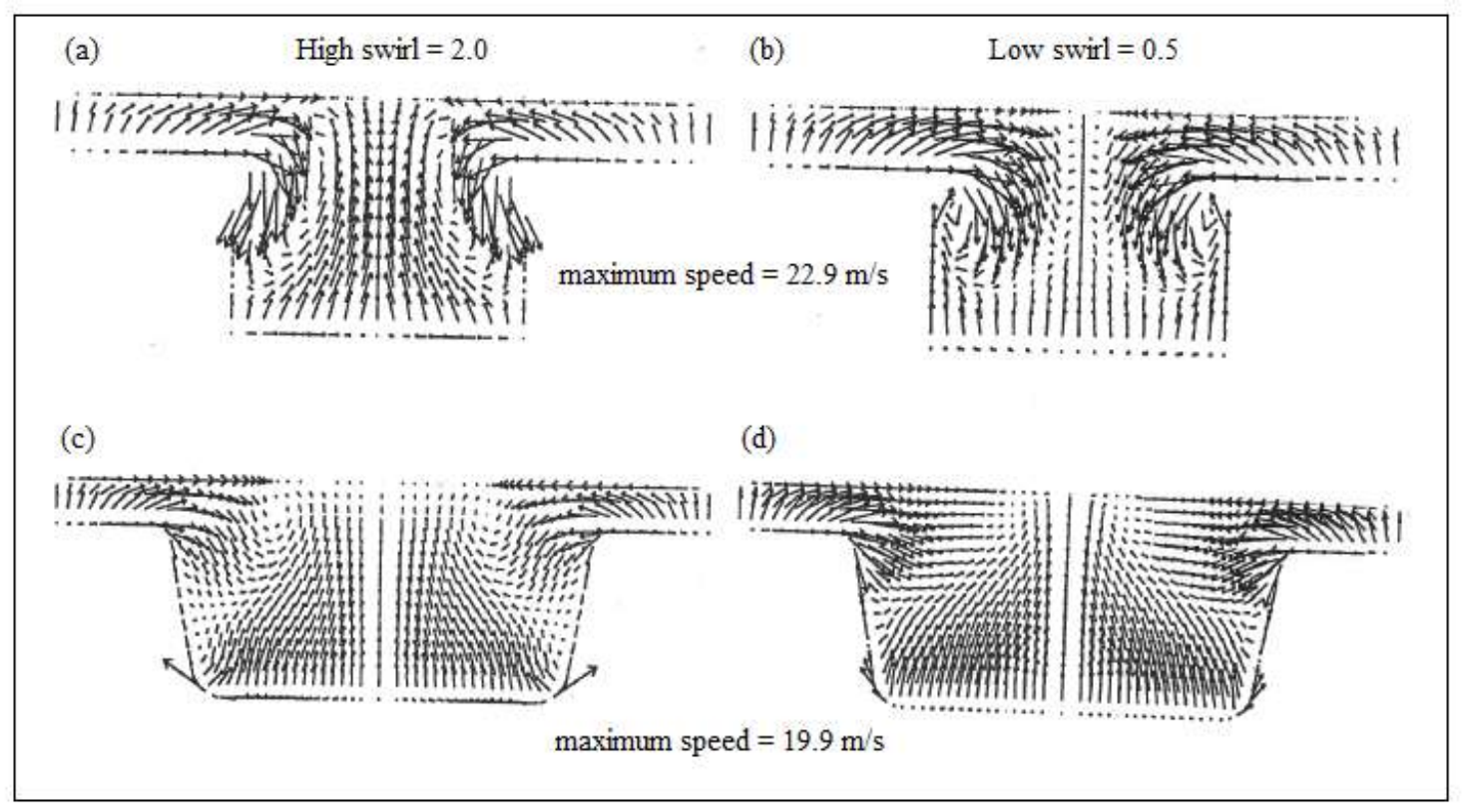

Fig. 4: Velocity vector plot across $J=1$ plane at 25 degree $B T D C$.

The observation raised above indicates that the re-entrant bowl configuration requires some form of optimization regarding global turbulence generation. One possible way of achieving this optimization is to design a reentrant combustion chamber which generates squish motions with zero re-attachment and longer penetration into the main stream flow. Where bowl modification is not feasible, then an optimal- level of intake swirl must be sought and used.

The squish motion generated by the bowl-in-piston configuration is not as intense as that generated by the reentrant bowl, but it does not re-attach itself to the chamber wall.Moreover, it has a longer penetration range than that prevailing in the re-entrant bowl. Because of its longer range it interacts more with the main stream flow and the result is the very noticeable vortex generated in the fourth quadrant. The vortex motion is very suitable for enhancing the mixing of air-fuel mixture and it also promotes uniform turbulence generation. The mixing is very important as it improves fuel economy and reduces emissions.

The figures also show the motion within the bowl-in-piston chamber leveling-off at the top dead center position. It can now be seen that the entire combustion chamber is now filled with uniformly distributed motion. 


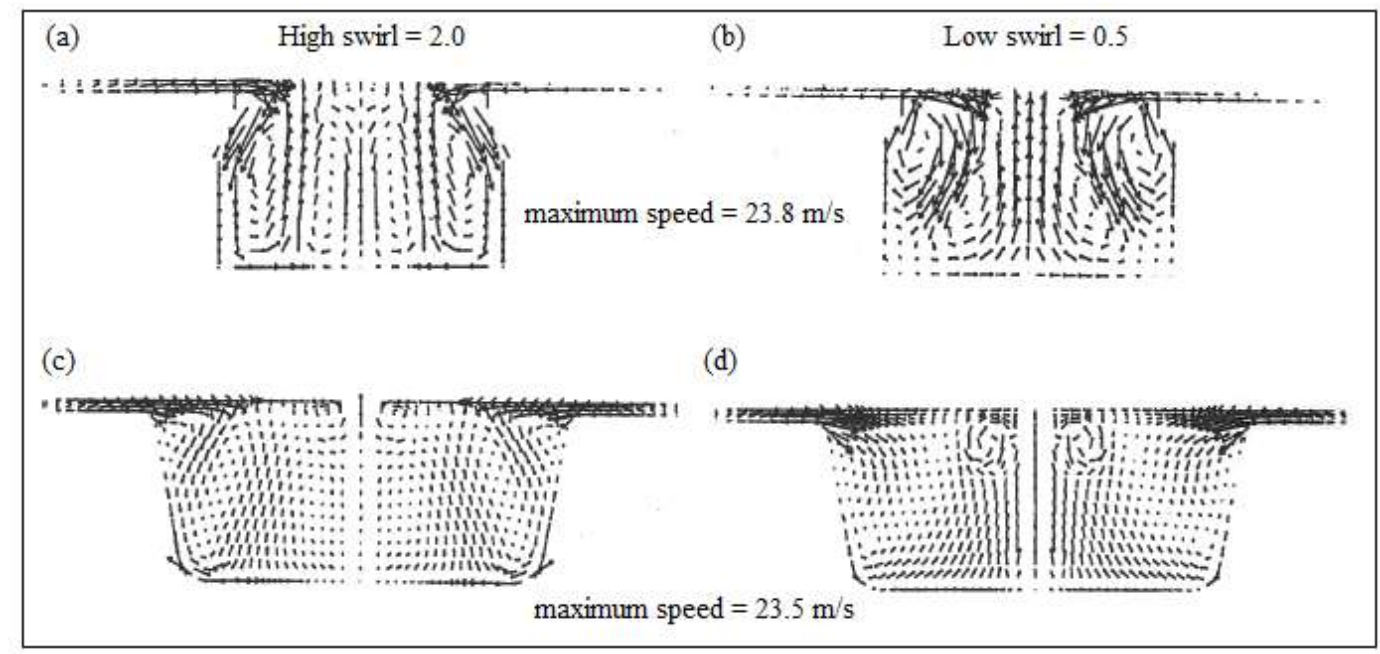

Fig. 5: Velocity vector plot across $J=1$ plane at 5 degree $B T D C$.

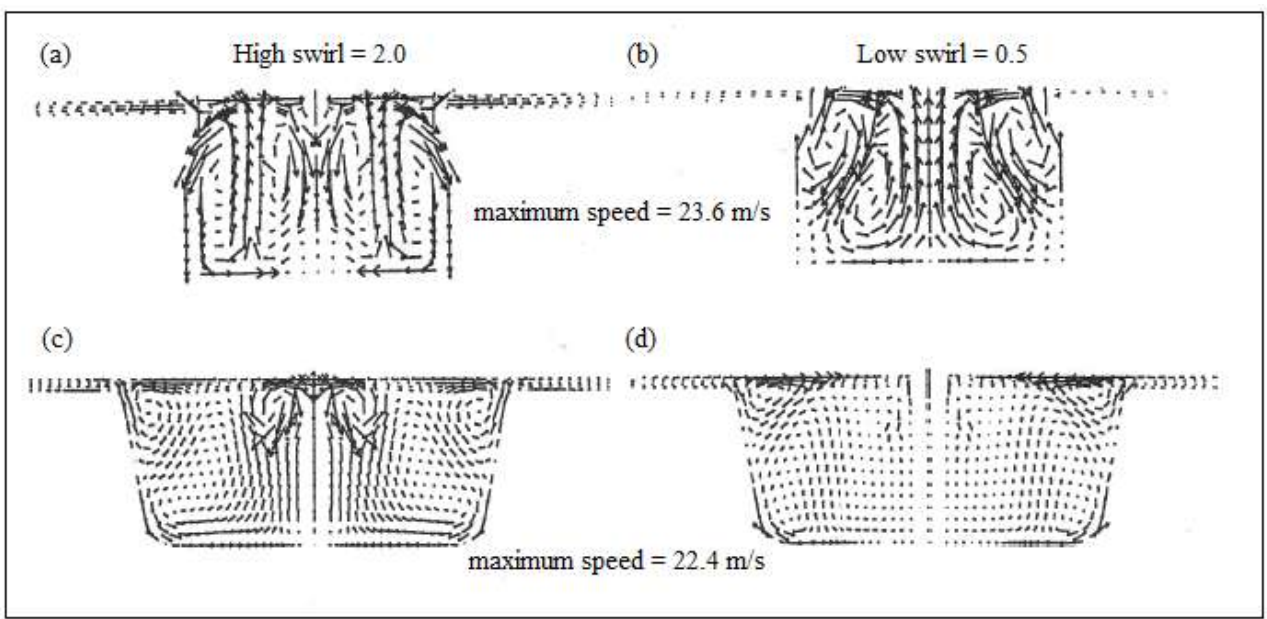

Fig. 6: Velocity vector plot across $J=1$ plane at $T D C$.

Figure 7 shows the reverse squish motion during the expansion stroke. The reverse squish motion is useful for scavenging operation as it helps clean the chamber prior to induction of fresh charge, thereby reducing charge dilution and consequent improvement in cycle-by-cycle variation.
Figure 8 shows the velocity vector plots at the $K$ plane, namely, $K=12$. Figs. $8 \mathrm{c}$ and $8 \mathrm{~d}$ show the pronounced effect of squish in the re-entrant bowl configuration. This is evident from the slight inclination of the velocityvectors.

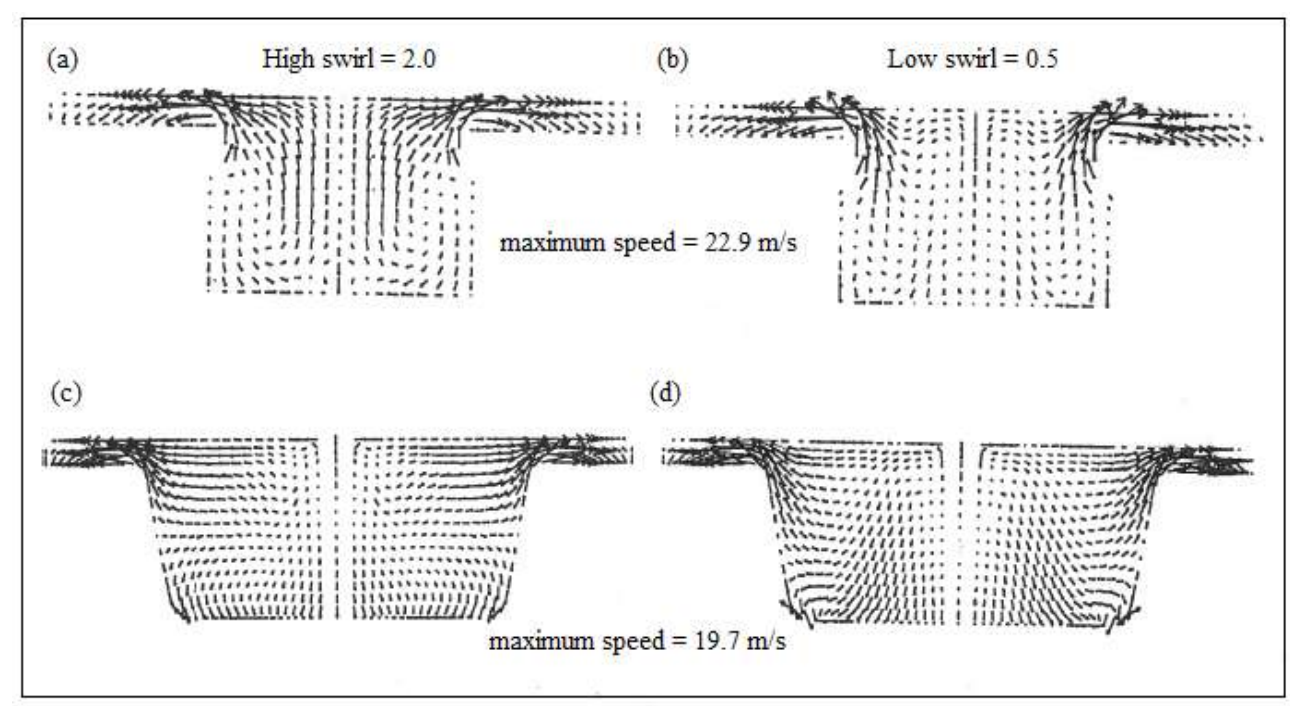

Fig. 7: Velocity vector plot across $J=1$ plane at 15 degree $A T D C$. 


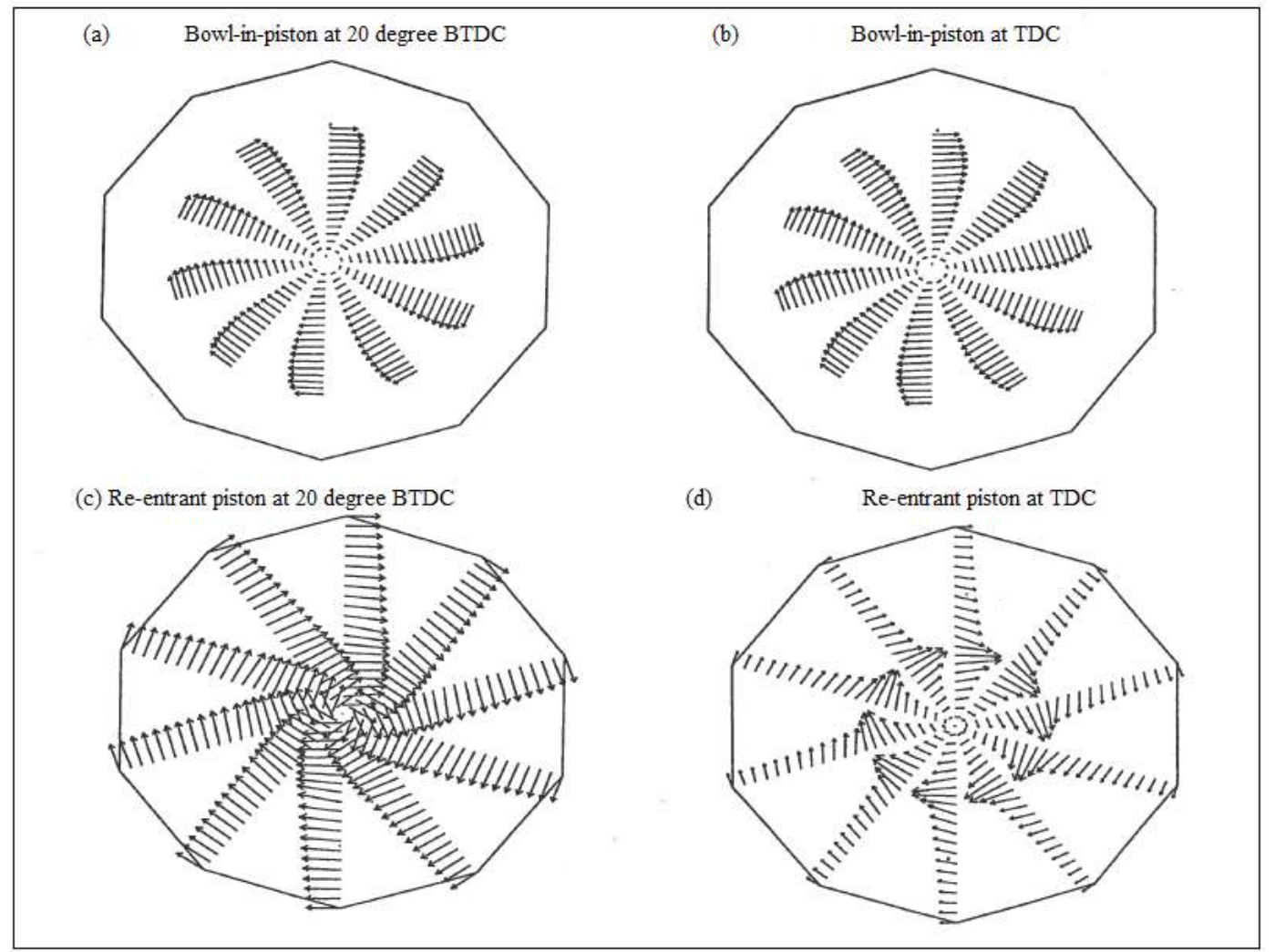

Fig. 8: Velocity vector plot across $K=12$ plane

\subsection{Turbulent Kinetic Energy}

The temporal distributions of total turbulence kinetic energy integrated over the relevant chamber volume from 20 degree $B T D C$ to the top dead center position are shown in Fig. 9. In each of the entries in Fig. 9, the contour line marked $H$ represents the maximum while that marked $L$ corresponds to the minimum. Figure 9 shows that the maximum values of the turbulent kinetic energy extend throughout the central region of the re-entrant bowl and are somewhat localized to interior portions of bowl-in-piston design throughout the compression stroke. Because of the shear flow induced by the squish motion, one might guess that the highest turbulence intensities would be observed near the rim of the piston bowl, however, the results show that the highest intensities are located in the interior of the piston bowl. This is because the dissipation rate of turbulent kinetic energy is inversely proportional to the length scale, $\ell$ through the relationship, $\varepsilon \sim k^{2 / 3} / \ell$, and $\ell$ is very small near walls. The observed high values of the turbulent kinetic energy around the lip of the re-entrant bowl design are due to strong squish actions. The figures show that the area covered by the maximum value of turbulence kinetic energy in the bowl-in-piston configuration is very small. This observation could be attributed to the relatively weaker squish generated by the bowl-in-piston configuration.

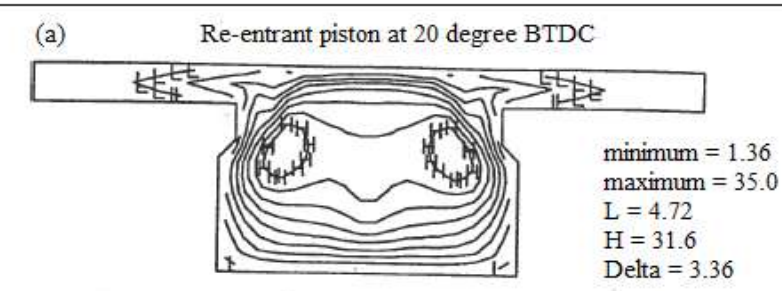

(c)

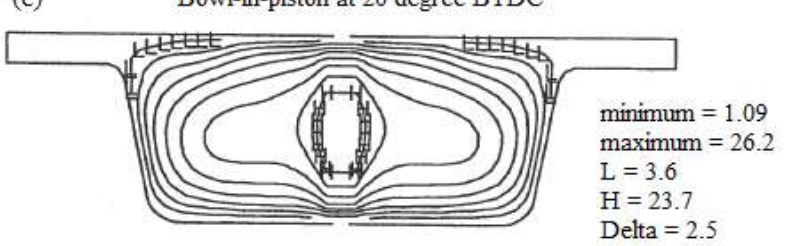

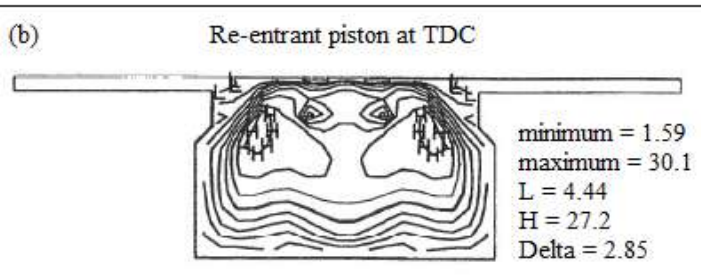

(d) Bowl-in-piston at TDC

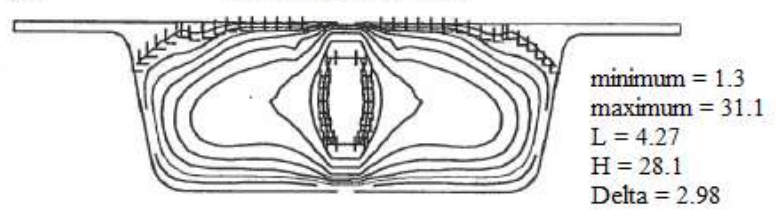

Fig. 9: Distribution of turrbulence kinetic energy $\left(\mathrm{m}^{2} / \mathrm{s}^{2}\right)$ across $J=1$ plane 
Figure $9 \mathrm{~b}$ and $9 \mathrm{~d}$ show the distribution of turbulent kinetic energy in both combustion chambers at the top dead center position. It was observed that the turbulence kinetic energy has virtually diminished to very low values everywhere within the combustion chambers except for the nugget of high turbulence kinetic energy located around the lip of the bowl in the re-entrant chamber design. Though the intake generated turbulence kinetic energy is almost completely dissipated by the action of viscous forces (diffusion), bowl geometries (particularly the re-entrant chamber configuration) was still able to enhance turbulence somewhat. A possible explanation for this could be due to the interaction of the stress field (squish) with the mean normal strain imposed by the piston motion and by compression of the induction generated vortex.

\subsection{Turbulent Length Scale}

The contours depicting the distribution of turbulent length scale from 20 degree $B T D C$ to the top dead center position are shown in Fig. 10. The profiles of turbulent length scale show that the maximum values corresponding to the $H$ contour lines occur at the center of all the combustion chambers. The lowest values of the turbulence length scale are distributed around the periphery of the chambers close to the walls. This is to be expected since turbulence length scales are determined by chamber geometry near top dead center with the integral scale of the order of the clearance gap in disc chambers and of bumping clearance in squish chambers. The magnitude of the turbulence length scale is a very important parameter as regards early flame development in the sense that it can be consumed very rapidly thereby resulting in the formation of a favorable flame kernel. The concentration of low values of the turbulence length scale around the lips of both the re-entrant- and the bowl-in-piston combustion chambers are consistent with the high levels of turbulence intensities generated by the action of squish in these areas.

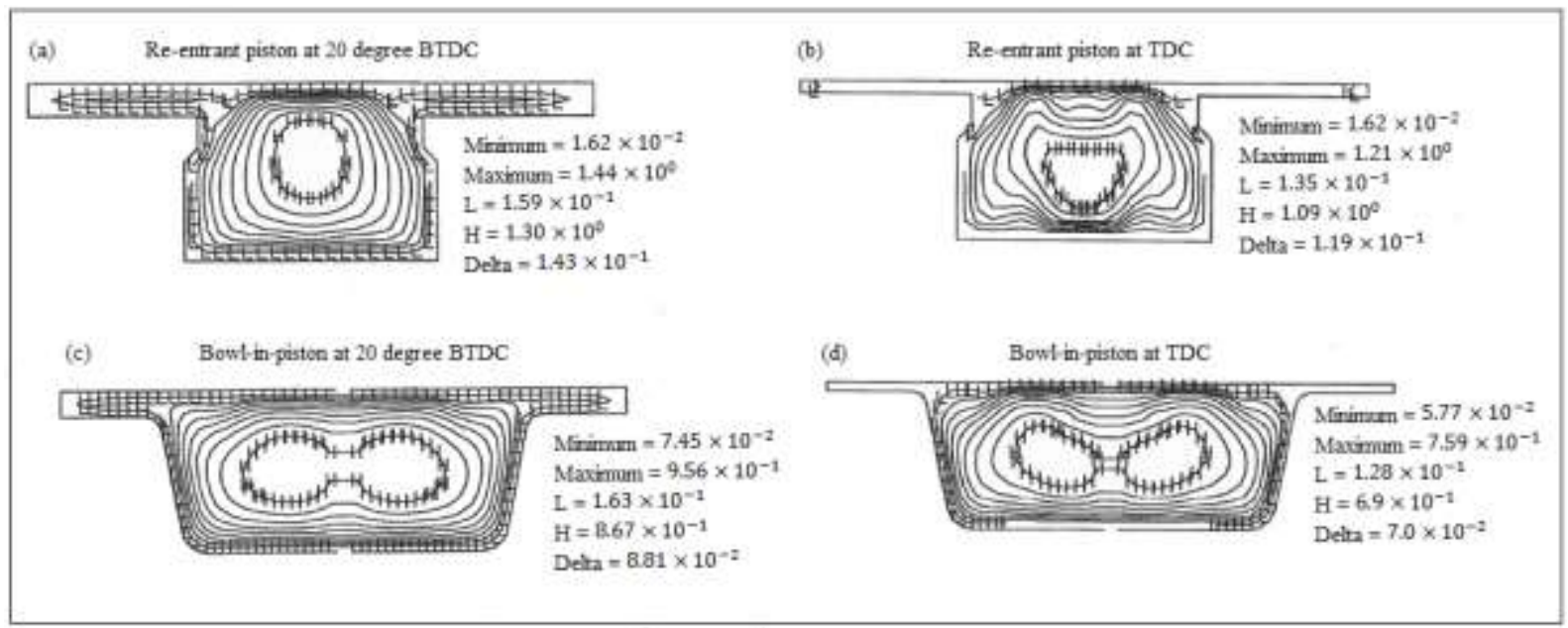

Fig. 10: Distribution of turrbulence length scale $(\mathrm{cm})$ across $J=1$ plane

\section{CONCLUSIONS}

The KIVA-II code was used to investigate the effects of reentrant and bowl-in-piston combustion chamber designs on salient flow and turbulence quantities. Based on the foregoing, the following conclusions were arrived at:

- The re-entrant bowl assembly generated 35\%more turbulence than the bow-in-piston design.

- The squish penetration for low intake swirl (0.5) was about 20\% greater than for high intake swirl (2.0).

- For both low and high intake swirl levels, the re-entrant bowl generated about 33\% more turbulence at top dead center than the bowl-in-piston configuration.

- In general, higher intake swirl values lead to higher flow and turbulence quantities.

- The flow fields generated were very informative and they were instrumental in visualizing trends which would have been impossible otherwise. Aprofound example was the flow re-attachment phenomenon depicted by the re-entrant bowl configuration. An immediate benefit that could be realized from this observation is to institute a program of chamber optimization.

- $\quad$ After top dead center, high shear and reverse-squish flow occurs in both combustion chambers, however, they were more intense in the re-entrant bowl assembly. This could be attributed directly to the reentrancy features of the bowl. This flow condition could enhance burn-up of unburned fuel with the concomitant reduction in hydrocarbon emissions.

\section{ACKNOWLEDGEMENTS}

The work reported herein was graciously supported by the Nigerian Defence Academy, Kaduna, Nigeria, Texas Southern University, Houston, Texas, USA and the Kwara State University, Malete, Nigeria. The authors also wish to express their sincere thanks to their colleagues and the reviewers for their insightful and constructive remarks which led to clarifying and improving this paper considerably. 


\section{NOMENCLATURE}

A

ATDC

$B T D C$

IVC

$n$

$Q$

TDC

$V_{\alpha}$

$y_{p}$

$A_{1}, A_{2}, A_{3}$

$c_{\varepsilon 1}, c_{\varepsilon 2}, c_{\varepsilon 3}$

$c_{\mu}$

Sc

$\operatorname{Pr}_{\varepsilon}$

$A_{0}$

$b_{x}, b_{y}$ and $b_{z}$

$c_{s}=1.5$

$D$

$\vec{F}^{s}$

$\vec{g}$

$\tilde{I}$

$\vec{i}$

$J_{1}$

$J_{2}$

$\vec{j}$

$k$

$\vec{k}$

$N_{E}$

$\vec{n}$

$p$

$P G S$

$\operatorname{Pr}_{k}$

$\vec{r}$

$R$

$r$

$R_{s}$

$c_{l w}, B, R_{c}$
Area of cell face

After Top Dead Center

Before Top Dead Center

Inlet Valve Closure

Cycle number

Dummy variable

Top Dead Center

Flux volumes calculated for cell $(i, j, k)$

Distance from the wall

Constants

Turbulence modeling constants

Empirical constant with a standard

value of 0.09

Schmidt number

Prandtl number in turbulent kinetic energy dissipation equation

Switch for selecting laminar or turbulent flow calculations

Components of the grid velocity $\vec{b}$

Standard turbulence modeling

constant

Diffusion coefficient

Rate of momentum gain per unit volume due to the spray

Specific body force

Unit dyadic

Unit vectors in $\mathrm{x}$ direction

First order Bessel Function

Bessel Function of order 2

Unit vectors in y direction

Turbulent kinetic energy

Unit vectors in $\mathrm{z}$ direction

Measured with a tachometer or read

from the engine manual

Unit normal vector to the wall

Fluid pressure

Pressure Gradient Scaling

Prandtl number in turbulent kinetic

energy equation

Position vector

Cylinder radius

Radial distance from the cylinder axis

Swirl ratio

Constants in the law-of-the-wall formulation

Time

$t$

$T$

$u^{*}$

$U_{t}$

$v$

$V_{i j k}$

$w(r)$

$w_{\text {wall }}$

$\dot{W}^{s}$

$x$

$y$

$z$

$\alpha$

$\Delta t^{n}$

$\delta_{i j}$

$\ell$

$\varepsilon$

$\kappa$

$\lambda_{1}$

$\lambda$

$\mu_{\text {air }}$

$\mu_{\text {eff }}$

$\nabla$

$v_{0}$

$\Omega_{E}$

$\omega_{s}$

$\rho$

$\tilde{\sigma}$

$\tilde{\sigma}_{w}$

$\tau$

$\xi$

$\rho_{m}$

$\rho^{s}$

\section{REFERENCES}

[1] Vehicle Technologies Office: Moving America Forward with Clean Vehicles, http://energy.gov/eere/vehicles/vehicle-technologies- 
office-moving-america-forward-clean-vehicles

(Accessed December 21, 2015).

[2] D. Clerk, Cylinder Actions in Gas and Gasoline Engines, SAE Journal, vol. 8, pp. 523-539, 1921.

[3] Y. X. Zhu, C. Savonen, N. L. Johnson, and A. A. Amsden, "Three-Dimensional Computations of the Scavenging Process in an Opposed-Piston Engine," SAE Technical Paper 941899, 1994.

[4] J. Miok, K. Y. Huh, S. H. Noh, and K. H. Choi, "Numerical Prediction of Charge Distribution in a Lean Burn Direct-Injection Spark Ignition Engine," SAE Technical Paper 970626, 1997.

[5] S. C. Kong and C.-W. Hong, "Multidimensional Intake Flow Modeling of a Four-Stroke Engine with Comparisons to Flow Velocity Measurements," SAE Technical Paper 970883, 1997.

[6] B. Dillies, A. Ducamin, L. Lebrere, and F. Neveu, "Direct Injection Diesel Engine Simulation: A Combined Numerical and Experimental Approach from Aerodynamics to Combustion," SAE Technical Paper 970880, 1997.

[7] J. L. Lumley, Engines - an introduction. Cambridge University Press, 1999

[8] A. Nauwerck, J. Gindele, U. Spicher, H. Rosskamp and G. Landwehr, Investigation of the transient In-Cylinder Flow Inside a Two Stroke Engine with Particle-ImageVelocimetry, SAE paper 2000-01-0902, 2000.

[9] E. Rouland, M. Trinite, F. Dionnet, A. Floch and A. Ahmed, Particle Image Velocimetry Measurements in a High Tumble Engine for In-Cylinder Flow Structure Analysis, SAE paper 972831, 1997.

[10] C. F. F. Rodriguez and J Mantilla, Modeling HCCI Engine Combustion Coupling Cantera to KIVA 4, SAE Technical Papers 04/2015; doi: 10.4271/2015-01-0816, 2015.

[11] S. Reifarth, Efficiency and Mixing Analysis of EGRSystems for Diesel Engines, Doctoral Thesis, Department of Machine Design, Royal Institute of Technology SE-100 44 Stockholm, 2014. ISBN 978-917501-997-0.

[12] P. S. Abraham, Analyzing in-cylinder Flow Variations in a Motored Spark Ignition Engine using Proper Orthogonal Decomposition, Ph.D. Thesis, Department of Mechanical Engineering, University of Michigan, 2013.

[13] H. W. Dembinski, The effects of injection pressure and swirl on in-cylinder flow pattern and combustion in a compression ignition engine, International Journal of Engine Research, 2013.

[14] M. K. Khair and H. Jääskeläinen, Exhaust Gas Recirculation, $\quad$ DieselNet, 072012. http://www.dieselnet.com/tech/engine_egr.php. (Accessed December 22, 2015).

[15] Y. Bakhshan., A. Shadaei and S. Niazi, QuasiDimensional Modeling of a CNG Fueled HCCI Engine Combustion Using Detailed Chemical Kinetic Advances in Chemical Engineering and Science, 2011, 1, 198-207. doi:10.4236/aces.2011.14029;

http://www.SciRP.org/journal/aces.

December 22, 2015).
[16] D. J. Torres and Trujillo, M. F., KIVA-4: An Unstructured ALE Code for Compressible Gas Flow with Sprays, Journal of Computational Physics, 291, 943-975, 2006

[17] A. A. Amsden, P. J. O'Rourke and T.D.Butler, KIVA-II: A Computer Program for Chemically Reactive Flows with Sprays, Technical Report LA 11560 MS, Los Alamos National Laboratory, 1989.

[18] S. V. Patankar, Numerical Heat Transfer and Fluid Flow. Taylor \& Francis, (1980). ISBN 978-0-89116522-4.

[19] A. A. Amsden, J. D. Ramshaw, P. J. O’Rourke and J. K. Dukowicz, KIVA: A Computer Program for Two- and Three-Dimensional Fluid Flows with Chemical Reactions and Fuel Sprays, Los Alamos National Laboratory, Report LA-10245-MS, 1985.

[20] R. G. Abdel-Gayed, K. J. Al-Khishali and D. Bradley, Turbulent Burning Velocities and Flame Straining in Explosions, Proc. R. Soc. London, vol. A391, pp. 393 414, 1984.

[21] G. E. Andrews, D. Bradley and S. B. Lwakabamba, Turbulence and Turbulent Flame Propagation - A Critical Appraisal, Combustion and Flame, 24, pp. 285304, 1975. 